\title{
Recent Advances in Genome Editing Using CRISPR/Cas9
}

\author{
Yuduan Ding ${ }^{1,2}$, Hong $\mathrm{Li}^{3}$, Ling-Ling Chen ${ }^{1,2 *}$ and Kabin Xie ${ }^{3 *}$ \\ ${ }^{1}$ National Key Laboratory of Crop Genetic Improvement, Huazhong Agricultural University, Wuhan, China, ${ }^{2}$ College of \\ Informatics, Huazhong Agricultural University, Wuhan, China, ${ }^{3}$ College of Plant Science and Technology, Huazhong \\ Agricultural University, Wuhan, China
}

The CRISPR (clustered regularly interspaced short palindromic repeat)-Cas9 (CRISPR-associated nuclease 9) system is a versatile tool for genome engineering that uses a guide RNA (gRNA) to target Cas9 to a specific sequence. This simple RNA-guided genome-editing technology has become a revolutionary tool in biology and has many innovative applications in different fields. In this review, we briefly introduce the Cas9-mediated genome-editing method, summarize the recent advances in CRISPR/Cas9 technology, and discuss their implications for plant research. To date, targeted gene knockout using the Cas9/gRNA system has been established in many plant species, and the targeting efficiency and capacity of Cas 9 has been improved by optimizing its expression and that of its gRNA. The CRISPR/Cas9 system

OPEN ACCESS

Edited by:

Basil J. Nikolau,

lowa State University, USA

Reviewed by:

Chung-Jui Tsai,

University of Georgia, USA

Yuriko Osakabe,

The University of Tokushima, Japan

*Correspondence:

Ling-Ling Chen

Ilchen@mail.hzau.edu.cn;

Kabin Xie

kabinxie@mail.hzau.edu.cn

Specialty section:

This article was submitted to

Technical Advances in Plant Science,

a section of the journal

Frontiers in Plant Science

Received: 19 January 2016 Accepted: 06 May 2016

Published: 24 May 2016

Citation:

Ding Y, Li H, Chen L-L and Xie K (2016) Recent Advances in Genome Editing Using CRISPR/Cas9.

Front. Plant Sci. 7:703. doi: 10.3389/fp/s.2016.00703 can also be used for sequence-specific mutagenesis/integration and transcriptional control of target genes. We also discuss off-target effects and the constraint that the protospacer-adjacent motif (PAM) puts on CRISPR/Cas9 genome engineering. To address these problems, a number of bioinformatic tools are available to help design specific gRNAs, and new Cas9 variants and orthologs with high fidelity and alternative PAM specificities have been engineered. Owing to these recent efforts, the CRISPR/Cas9 system is becoming a revolutionary and flexible tool for genome engineering. Adoption of the CRISPR/Cas9 technology in plant research would enable the investigation of plant biology at an unprecedented depth and create innovative applications in precise crop breeding.

Keywords: CRISPR/Cas9, plants, genome editing, guide RNA, bioinformatic tools

\section{INTRODUCTION}

DNA sequencing technology has enabled us to decipher the sequence of whole genomes and to profile the transcriptomes of many organisms with unprecedented throughput, scalability, speed, and low cost. The knowledge of plant genomes and transcriptomes is increasing every day, opening the possibility of creating new crop cultivars through molecular design and genetic engineering. However, our ability to precisely modify plant genomes used to be limited until the emergence of efficient genome-editing technology. This technology uses designer nucleases and the cellular DNA repair system to precisely modify genomic sequence (Voytas, 2013). In practice, a synthetic sequence-specific nuclease is designed to recognize the chosen genomic site and is transfected into the cell, where it creates a double-strand DNA break (DSB) at the site. This DSB is usually repaired by the endogenous, error-prone non-homologous end joining (NHEJ) pathway, which introduces a 
small insertion or deletion (InDel) at the site, thus knocking out the gene. If a donor DNA fragment with homology to the flanking sequence is present, as with gene knock-in experiments, the DSB is repaired through homology-directed repair (HDR) using the donor DNA as a template. As a result, the sequence of the donor fragment is integrated into the genome at the DSB site. The DSB repair system (NHEJ and HDR) is a ubiquitous component of all living cells; therefore, an artificial nuclease whose recognition site is reprogrammable is the most critical part of genome editing.

To date, three programmable nucleases have been developed for genome editing, including zinc finger nucleases (ZFNs; Pabo et al., 2001), transcription activator-like effector nucleases (TALENs; Boch et al., 2009; Moscou and Bogdanove, 2009), and RNA-guided nucleases (RGNs) from the clustered regularly interspaced short palindromic repeat (CRISPR) and CRISPRassociated proteins (Cas) system (van der Oost, 2013). Among these nucleases, the Cas9 nuclease, which recognizes target DNA according to Watson-Crick base pairing between its guide $\mathrm{RNA}(\mathrm{s})$ and DNA, is the simplest one to implement and quickly became the most popular and powerful tool for genome engineering. The advanced CRISPR/Cas9 technology not only provides a molecular tool for investigating biological questions in depth, but also enables the development of innovative and practical applications of biology (Pennisi, 2013; Doudna and Charpentier, 2014; Hsu et al., 2014).

The CRISPR/Cas9 technology is a modern, fashionable method in plant research. Immediately after its early use to edit the genomes of animals and bacteria (Cong et al., 2013; Hwang et al., 2013; Jiang et al., 2013a; Mali et al., 2013b), its efficacy was demonstrated in the model plant systems of Arabidopsis, rice, sorghum, and tobacco (Feng et al., 2013; Jiang et al., 2013b; Li et al., 2013; Mao et al., 2013; Miao et al., 2013; Nekrasov et al., 2013; Shan et al., 2013; Xie and Yang, 2013). Nowadays, this technology is broadly used in different plant species, and dozens of CRISPR/Cas9 vectors are available in the public plasmid repository of Addgene (http://www.addgene.org/crispr/plant/). The CRISPR/Cas9 pioneers have improved this system into a flexible and powerful platform for genome engineering. In this review, we briefly summarize the recent advances of CRISPR/Cas9 technology and its impact for plant genome engineering.

\section{SEQUENCE-SPECIFIC DNA TARGETING WITH THE CRISPR/Cas9 SYSTEM}

The CRISPR/Cas9 system comes from the adaptive immune system of bacteria and archaea, which detects and degrades invasive DNA from bacteriophages and plasmids (Fineran and Charpentier, 2012). The most commonly used RGN in genome editing is the Cas9 nuclease from the type II CRISPR/Cas9 system of Streptococcus pyogenes (Gasiunas et al., 2012; Jinek et al., 2012). In the prototype of the CRISPR/Cas9 system, Cas9 was directed to the DNA target by an RNA duplex of crRNA and tracrRNA, but a single guide RNA (gRNA) is used instead for genome editing (Gasiunas et al., 2012; Jinek et al., 2012, 2013; Cong et al., 2013; Mali et al., 2013b). As shown in Figure 1, there are three requirements for Cas9-mediated genome editing: (1) a Cas9 protein with a nuclear localization signal; (2) a gRNA consisting of a guide sequence (referred to as the gRNA protospacer, 20 nt) at the $5^{\prime}$-end that matches the DNA sequence of the target site and a conserved $3^{\prime}$-end scaffold with a special stem-loop structure that binds Cas9; (3) a protospacer-adjacent motif (PAM, sequence of $5^{\prime}$-NGG- $3^{\prime}$ ) in the genomic sequence downstream of the targeted DNA. In theory, any genomic sequence bearing a PAM could be edited by Cas9 with a specific gRNA. Owing to the high occurrence of PAMs in genomes, Cas9/gRNA can target almost every gene.

Cas9-mediated plant genome editing can be easily implemented using plasmid vectors containing the Cas9 and gRNA expression cassettes. Normally, a DNA-dependent RNA polymerase II (Pol II) promoter and a Pol II transcriptional terminator are used to express Cas 9 fused with a nuclear localization signal peptide, and a Pol III promoter and terminator is used to express the gRNA. The Cas9 and gRNA expression cassettes are often put in one plasmid, which is then delivered into plant cells using conventional transformation methods. Alternatively, one can microinject or transfect in vitro-synthesized Cas9 mRNA (or protein) and gRNA(s) into animal embryos (Kim et al., 2014; Ramakrishna et al., 2014) and plant protoplasts (Woo et al., 2015). This DNA-free genome-editing approach has sparked new breeding technologies based on CRISPR/Cas9. However, because the regeneration capacity of protoplasts is very low for most plant species, the direct injection method only suits few plants. In addition to targeted genome editing, the CRISPR/Cas9 system is also a versatile platform to manipulate genomes for different purposes.

\section{TARGETED GENE KNOCKOUT USING Cas9/gRNA IN PLANTS}

The functional knockout of a target gene is a basic step in plant genetic analysis. In principle, it could be readily obtained using Cas9/gRNA to introduce InDels into the coding region, which will disrupt translation (Figure 1). Indeed, the Cas9/gRNA method has been successfully used to create gene knockouts in Arabidopsis, rice, tobacco, and sorghum (Kuhn and Binder, 2002; Feng et al., 2013; Li et al., 2013; Mao et al., 2013; Miao et al., 2013; Nekrasov et al., 2013; Shan et al., 2013; Xie and Yang, 2013). These early proof-of-concept studies proved that Cas9/gRNA generates InDels at target sites and functionally knocks out target genes with variable efficiency. Further analysis found that the edited gene faithfully passed on to the next generation of Arabidopsis (Feng et al., 2014) and rice (Zhang et al., 2014). In the past 3 years, CRISPR/Cas9-mediated genome editing has been established in many important crops including maize (Liang et al., 2014; Svitashev et al., 2015), wheat (Wang et al., 2014), barley (Lawrenson et al., 2015), soybean (Jacobs et al., 2015; Li et al., 2015), tomato (Brooks et al., 2014), sweet orange (Jia and Wang, 2014), petunia (Zhang et al., 2016), rapeseed (Lawrenson et al., 2015), and poplar (Fan et al., 2015; Zhou et al., 2015). The 


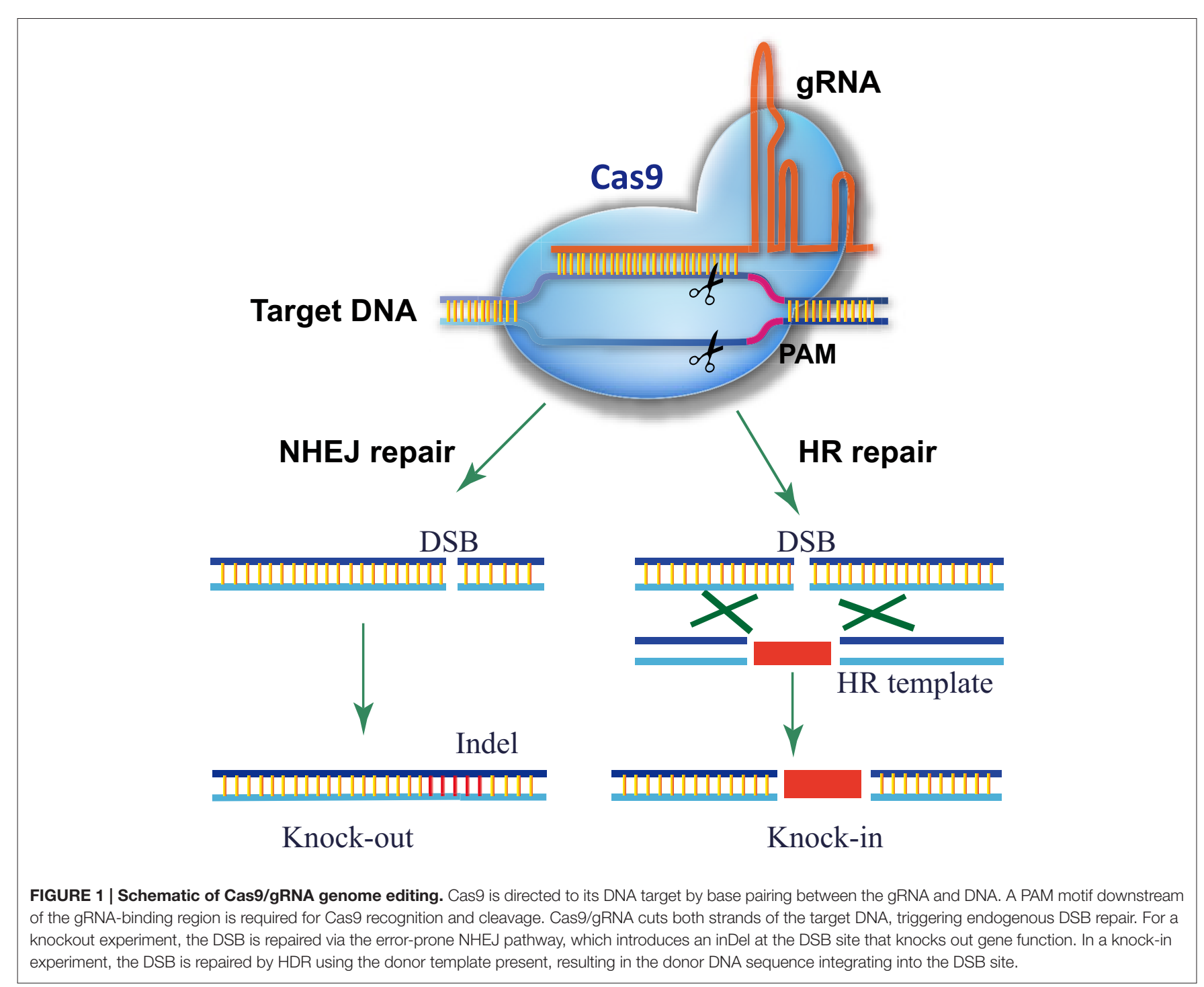

Cas9/gRNA system has become the primary choice for creating knockout mutants in plants.

In these early plant genome-editing experiments, Cas9/gRNA had a higher knockout frequency in rice than in Arabidopsis. Bi-allelic mutations were frequently detected in the T0 generation of genome-edited rice lines; however, most Arabidopsis T1 lines carried somatic mutations and required several generations to obtain the knockout mutants. For example, in phytoene desaturase 3 (PDS3) knockout experiments, which evaluated knockout efficiency in plants, the albino phenotype of the $p d s 3$ null mutant was observed in rice (Shan et al., 2013), but not in Arabidopsis (Li et al., 2013; Nekrasov et al., 2013), in the primary generation of transgenic plants. It is likely that DSB repair capacity differs across recipient cell types, affecting editing efficiency and/or the timing of InDel formation during the transformation process.

This phenomenon prompted researchers to optimize Cas9 expression in Arabidopsis using cell- and tissue-specific promoters. In comparison to the previous constitutive $35 \mathrm{~S}$ promoter, Cas9/gRNA editing efficiency increased after expressing Cas9 under different promoters such as the dividing cell-specific INCURVATA2 promoter (Hyun et al., 2015), various egg cell-specific promoters (Wang et al., 2015), the cell division-specific YAO promoter (Yan et al., 2015), and the germ-line-specific SPOROCYTELESS promoter (Mao et al., 2016). These successful examples imply that the spatiotemporal expression of Cas9/gRNA is important for highly efficient genome editing in Arabidopsis. Because the genome editing outcome is also dependent on the endogenous DSB repair machinery, further elucidation of the DNA repair mechanism would facilitate development of more efficient genome-editing tools based on Cas9/gRNA.

The capacity of the gRNA cassette is critical for multiplex genome editing. However, the promoter used to express a single gRNA in plants has more restrictions than the Cas9 promoter. This is because the gRNA is a small non-coding RNA and 
requires an accurate $5^{\prime}$-end to keep its target-specific spacer sequence. In Cas9 genome editing, the most commonly used promoters are the snoRNA U3 and U6 genes from different plant species. To simultaneously knock out multiple genes, different gRNA expression cassettes can be inserted into one plasmid, thus guiding Cas9 to different targets (Xing et al., 2014; Zhou et al., 2014; Lowder et al., 2015; Ma et al., 2015). However, transcripts from U3 and U6 promoters are obligated to start with the nucleotides "A" and "G," respectively. Such a restriction reduces the targeting range and potentially the efficiency of Cas9. Alternative ways to express gRNA have been developed, which enable more flexible and robust gRNA expression than did the original U3 and U6 promoters. In these, the gRNA is fused with an RNA fragment, and engineered ribonucleases excise the gRNAs from the transcript inside the cell. Three RNA processing systems have been engineered for gRNA processing: self-cleaving ribozyme (Gao and Zhao, 2014), the ribonuclease Csy4 (Nissim et al., 2014), and the endogenous transfer RNA (tRNA) processing system (Xie et al., 2015). Csy4 is an RNA endonuclease from the CRISPR/Cas system in Pseudomonas aeruginosa and specifically binds and cleaves a 28-nt RNA sequence (Qi et al., 2012). When this RNA sequence flanks gRNAs, Csy4 will cleave out the gRNA. After optimization to enhance the expression of this system, the Csy4 method now has efficient gRNA expression and processing for multiplex genome editing in human cells (Nissim et al., 2014), but its efficacy in plants requires further study. A strategy for robust expression of many different gRNAs in plants, as well as potentially all living organisms, is hijacking the tRNA processing system. The primary transcript of tRNA is precisely cut at both ends by endogenous RNases. Because the tRNA structure is sufficient for recognition and cleavage by these RNases, artificial genes with polycistronic tRNA-gRNA could be used for multiplex genome editing. An additional benefit is that tRNA contains an intragenic cis-element for Pol III transcription; thus tRNA can also be used as a transcriptional enhancer for the expression of polycistronic tRNA-gRNA gene. Indeed, the tRNA-gRNA method boosts Cas9 targeting capability (Xie et al., 2015).

After 3 years of development, a fruitful CRISPR/Cas9 toolbox is available for targeted gene knockout in plants. Particularly, multiplex gRNA expression enables the development of innovative applications that benefit genetic analysis (Figure 2). This toolbox not only enables the knockout of coding genes, but also allows the study of non-coding elements, which have been a challenge in plant genetic analysis (Figure 2). For example, microRNA genes could be knocked out by introducing InDels at the mature sequence if a PAM is available, thus disturbing the function of the mature microRNA. Alternatively, short microRNA fragments could be deleted using Cas9 and multiple gRNAs. Thus, microRNA gene and other non-coding elements

\section{Knock-out with Cas9/gRNA}

Introduce indels within the functional elements (eg., cis-element of promoter, mature miRNA sequence).

Use Cas 9 and a pair of gRNAs to delete a target non-coding fragment.

Non-coding fragment non-coding RNA gene Promoter 5'-UTR
Introduce indels that damage the mRNA translation.

Delete a fragment from the coding sequence.

Mutate the intron-exon junction to generate a splicing variant.

Protein coding fragment

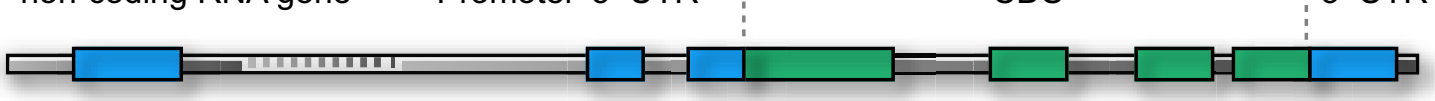

\section{Knock-in with Cas9/gRNA}

Insert a reporter to track protein expression or location.

Mutate specific amino acids to change protein activity.

Introduce foreign DNA fragments to confer new traits.

FIGURE 2 | Potential applications of CRISPR/Cas9 genome editing in plant genetic analysis. 
(e.g., cis-elements of promoters, enhancers, and transposons) can be knocked out (Figure 2). This proposed method is supported by the fact that the Cas9/gRNA system can generate stable plant lines with deletions of chromosomal fragments (Zhou et al., 2014; Xie et al., 2015). Owing to its simplicity and low cost, the Cas9/gRNA system can be used to generate knockout libraries covering the whole genome or a specific set of genes. Such genome-edited lines would not only give insights into plant biology, but also provide valuable genetic materials for crop breeding.

\section{Cas9-MEDIATED SITE-DIRECTED MUTAGENESIS AND KNOCK-IN IN PLANT GENOMES}

Harnessing the site-directed mutagenesis and site-specific integration of a gene (a knock-in) is of great value in precision crop breeding. In this procedure, HDR repair of DSBs using a supplied DNA template results in the donor sequence (e.g., an herbicide resistance gene) being substituted into the specified region (Figure 1). Cas9/gRNA-mediated sitedirected mutagenesis and knock-in has been used in rice and Arabidopsis protoplasts ( $\mathrm{Li}$ et al., 2013; Shan et al., 2013). Most recently, CRISPR/Cas9 was successfully used to introduce two substitutions in an acetolactate synthase gene in rice, which confers resistance to the herbicide bispyribac sodium (Sun et al., 2016). However, generating stable knock-in plants is still a challenge for most plant species because of several technical challenges. A donor DNA template must be codelivered into recipient cells, which increases the complexity of the experiment. Moreover, NHEJ occurs at a much higher frequency than HDR during DSB repair (Ray and Langer, 2002); thus it requires additional selection markers and more labor to identify the real knock-in lines from the other transformed plants. Among the efforts to overcome these technical challenges, a remarkable method is using the plant DNA virus replicon as the donor template (Baltes et al., 2014). The high copy number of donor DNA in geminivirus replicons greatly increases knock-in efficiency in plants. Nevertheless, CRISPR/Cas9 provides a simple method to generate a DSB at a target site to trigger HDR repair, but further optimization is still required to increase the efficiency of site-directed mutagenesis and knock-in for plant genome editing.

\section{Cas9-MEDIATED TRANSCRIPTIONAL REGULATION OF TARGET GENES}

Cas9 is not only a molecular scissor to edit genomic sequence, but also a versatile platform for delivering different regulatory components to a specific site. After removing the catalytic activity of Cas9 through D10A and H804A substitutions (dCas9), dCas9 and gRNA can be used as a shuttle for carrying different cargos to the DNA targets. dCas9 was engineered as a powerful tool for different purposes, particularly for transcriptional control of target genes. Early experiments in animals suggest that dCas9 fused with a transcriptional activation domain (AD, e.g., VP64) and guided by gRNA can activate the expression of the target gene (Figure 3), but 2-5 gRNAs are required to simultaneously target one promoter for robust transcriptional activation (Cheng et al., 2013; Gilbert et al., 2013; Maeder et al., 2013; Perez-Pinera et al., 2013; Chavez et al., 2015).

A more delicate strategy is using the gRNA scaffold as the platform for carrying transcriptional regulatory components to the genomic targets (Figure 3). This strategy integrates an RNA ligand and binding protein (e.g., MS2 and MCP [MS2 coat protein]) into the dCas9/gRNA system, creating a threecomponent system (Konermann et al., 2015; Zalatan et al., 2015). In this system, a specific RNA ligand (MS2) is added into the loop or $3^{\prime}$-end of the gRNA, without impairing its binding with Cas9,

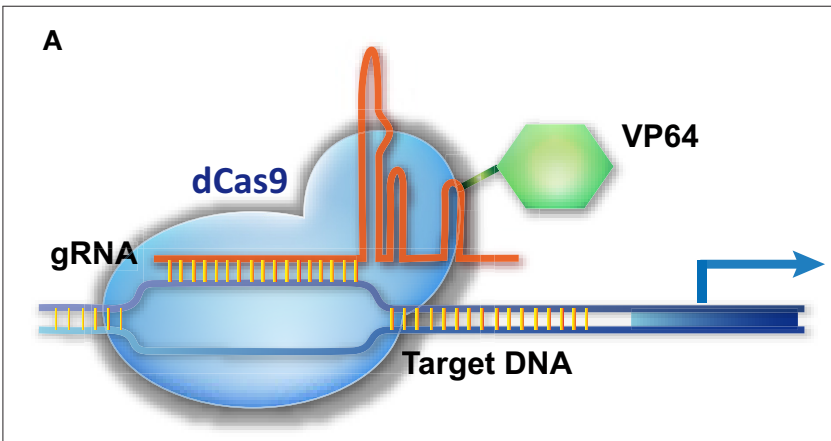

B

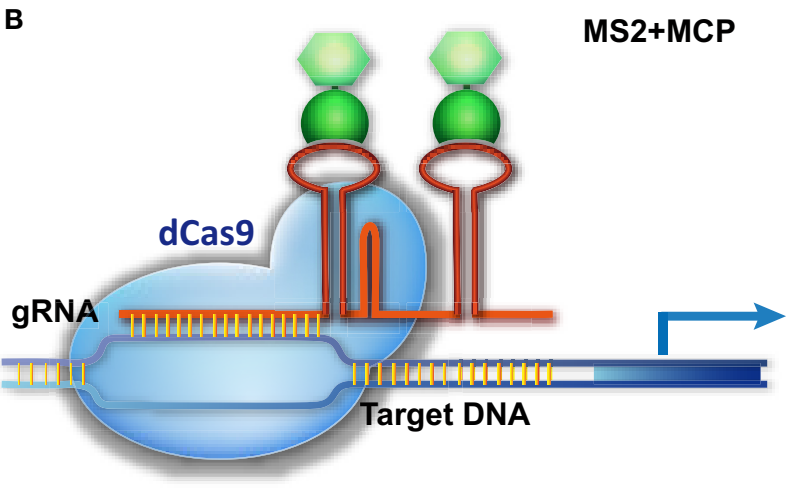

C

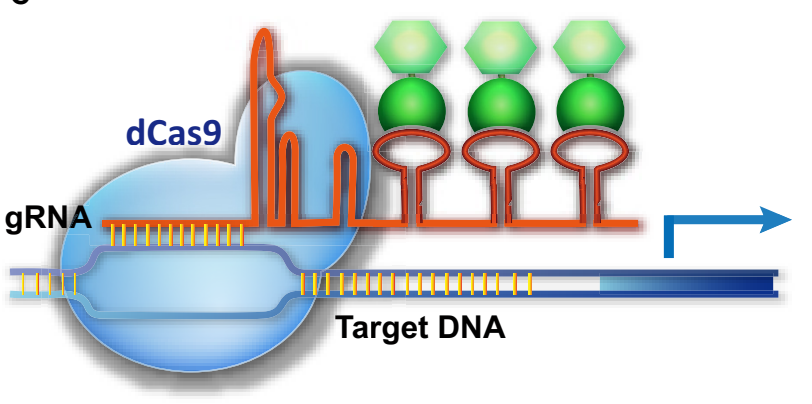

FIGURE 3 | CRISPR/Cas9-based transcriptional activation of target genes. (A) The dCas9-AD is guided to the promoter of a target gene, activating transcription. (B,C) A three-component system for target gene activation. The gRNA is modified by adding an RNA ligand, MS2, to its loop or $3^{\prime}$-end, without impairing its binding to dCas9. MCP, which specifically binds the MS2 ligand, is fused with the AD fragment. The dCas9/gRNA-MS2 is directed to the promoter of the target gene and recruits MCP-AD to activate transcription. 
and the RNA ligand-binding protein (MCP) is fused with the $\mathrm{AD}$ domain. As a result, the dCas9/gRNA-MS2 recruits MCP$\mathrm{AD}$ to its DNA target for transcriptional activation (Figure 3). In this system, multiple MS2 molecules could be added to the gRNA scaffold to simultaneously recruit several MCP-AD effector proteins for robust transcriptional activation (Figure 3). As a result, a single modified gRNA is sufficient for activating one gene (Konermann et al., 2015; Zalatan et al., 2015). The $\mathrm{AD}$ domain can be replaced by a transcriptional suppressor or other DNA-modifying enzyme to manipulate gene transcription (Zalatan et al., 2015) and epigenetic status (Hilton et al., 2015), respectively. Together with multiple gRNA-expressing devices, this three-component system can be used to execute complicated transcriptional programs to modulate cell activity.

In plants, target gene activation and suppression using dCas9 and gRNA was verified using the agroinfiltration transient expression system in Nicotiana benthamiana leaves (Piatek et al., 2015). However, similar to human cells, multiple gRNAs are required to target one promoter for robust activation or suppression. The three-component system of dCas9/gRNA-MS2/MCP-AD should enable more robust transcriptional control in plants, though additional optimization might be required. The transcriptional reprogramming capacity of dCas9/gRNA would enable us to modulate a quantitative trait and to redirect the metabolome to produce valuable nutrients or bio-agents through controlling the expression of multiple genes in plants.

\section{THE OFF-TARGET ACTIVITY OF Cas9/gRNA IN GENOME EDITING}

The off-target activity of Cas9 used to be a major concern as it can edit a DNA target bearing as many as five mismatches to its gRNA (Cradick et al., 2013; Fu et al., 2013). This off-target effect has been thoroughly analyzed by different in vitro and in vivo approaches (Hsu et al., 2013; Mali et al., 2013a; Pattanayak et al., 2013), and the fidelity of Cas9/gRNA can be summarized as follows: (1) In most cases, Cas9/gRNA cannot recognize a DNA site bearing more than three mismatches; (2) Cas9/gRNA cannot recognize and edit a DNA site with any number of mismatches near a PAM (within 10-12 bp); (3) The higher the concentration of Cas9/gRNA, the greater the potential for off-target effects; (4) Some $5^{\prime}-\mathrm{NAG}-3^{\prime}$ PAM sites can be targeted by Cas9/gRNA in bacteria and in in vitro experiments, but Cas 9 has much less affinity for NAG-PAM than for NGG-PAM. Furthermore, methods based on next-generation sequencing, such as GUIDEseq (Tsai et al., 2015), Digenome-seq (Kim et al., 2015), and ChIP-seq (Kuscu et al., 2014), can identify off-target sites for Cas9/gRNA. These high-throughput analyses also confirmed that Cas 9 has off-target activity and gRNA design is essential to reduce Cas9 off-targeting risk (bioinformatic tools can help determine this, see below).

The off-target editing of Cas9 has also been observed in rice protoplasts (Xie and Yang, 2013). However, a genome-wide survey of gRNA design revealed that a sufficient number of highly

TABLE 1 | Cas9 variants and orthologs for genome editing.

\begin{tabular}{lll}
\hline Cas9 (species) & PAM sequence $\left(\mathbf{5}^{\prime}->\mathbf{3}^{\prime}\right)$ & References \\
\hline Cas9 (STREPTOCOCCUS PYOGENES) & & \\
Cas9 wild type & NGG & Cong et al., 2013; Hwang et al., 2013; Ran et al., 2013b \\
Cas9 D1135E & NGG (reduced NAG binding) & Kleinstiver et al., 2015b \\
Cas9 37R3-2 (37R3-2 intein inserted & NGG (higher specificity) & Davis et al., 2015
\end{tabular}

into Cas9)

Cas9 (N497A-R661A-Q695A-Q926A)

Cas9 VRER variant

Cas9 EQR variant

Cas9 VQR variant

Cas9

(N497A/R661A/Q695A/Q926A), also

referred to as Cas9-HF1

Cas9 (K810A/K1003A/R1060A), also

referred to as eSpCas9 (1.0)

Cas9 (K848A-K1003A-R1060A), also

referred as eSpCas9 (1.1)

\section{Cas9 ORTHOLOGS}

SaCas9 (Staphylococcus aureus)

SaCas9 KKH variant

SaCas9

FnCas9 variant (Francisella novicida)

Cpf1 (Francisella novicida)

StCas9

NmCas9 (Neisseria meningitides)

StCas9 (Streptococcus thermophiles)
NGG (no detectable off-target effects)

NGCG

NGAG

NGAN or NGNG

NGG (no detectable off-target effects)

NGG (reduces off-target effects and maintains robust on-target cleavage)

NGG (no detectable off-target effects)
Kleinstiver et al., 2015b

Kleinstiver et al., 2015b

Kleinstiver et al., 2015b

Kleinstiver et al., 2016

Slaymaker et al., 2016

Slaymaker et al., 2016
NNGRRT or NNGRR(N)

NNNRRT

NAG or NGA or NNGGGT

$Y G$

TTN

NNAGAA or NNGGAA

NNNNGATT

NNAGAAW
Kleinstiver et al., 2015b; Ran et al., 2015

Kleinstiver et al., $2015 \mathrm{a}$

Kleinstiver et al., 2015b; Steinert et al., 2015

Hirano et al., 2016

Ran et al., 2015

Kleinstiver et al., 2015b; Steinert et al., 2015

Hou et al., 2013; Lee et al., 2016

Deveau et al., 2008 


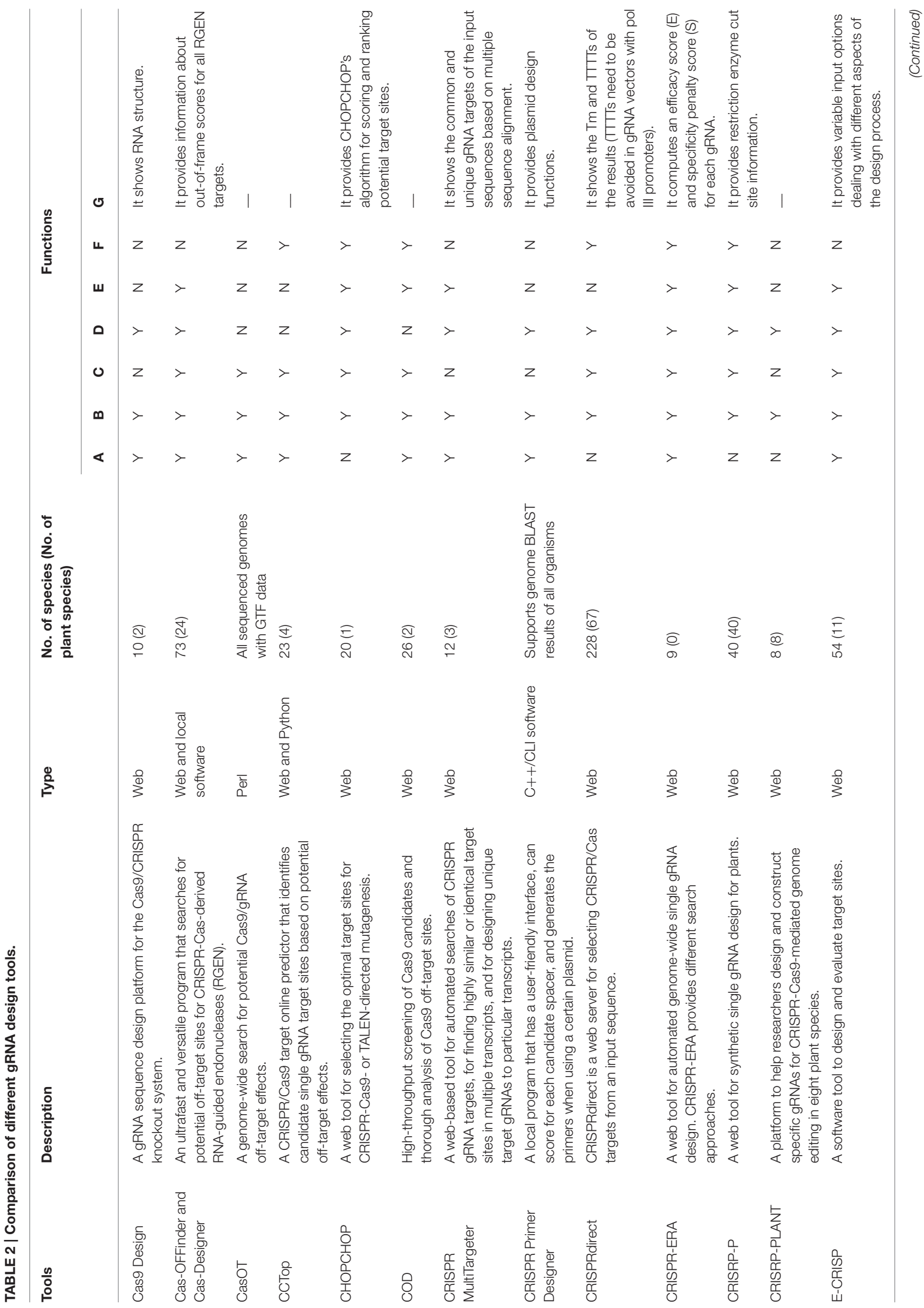




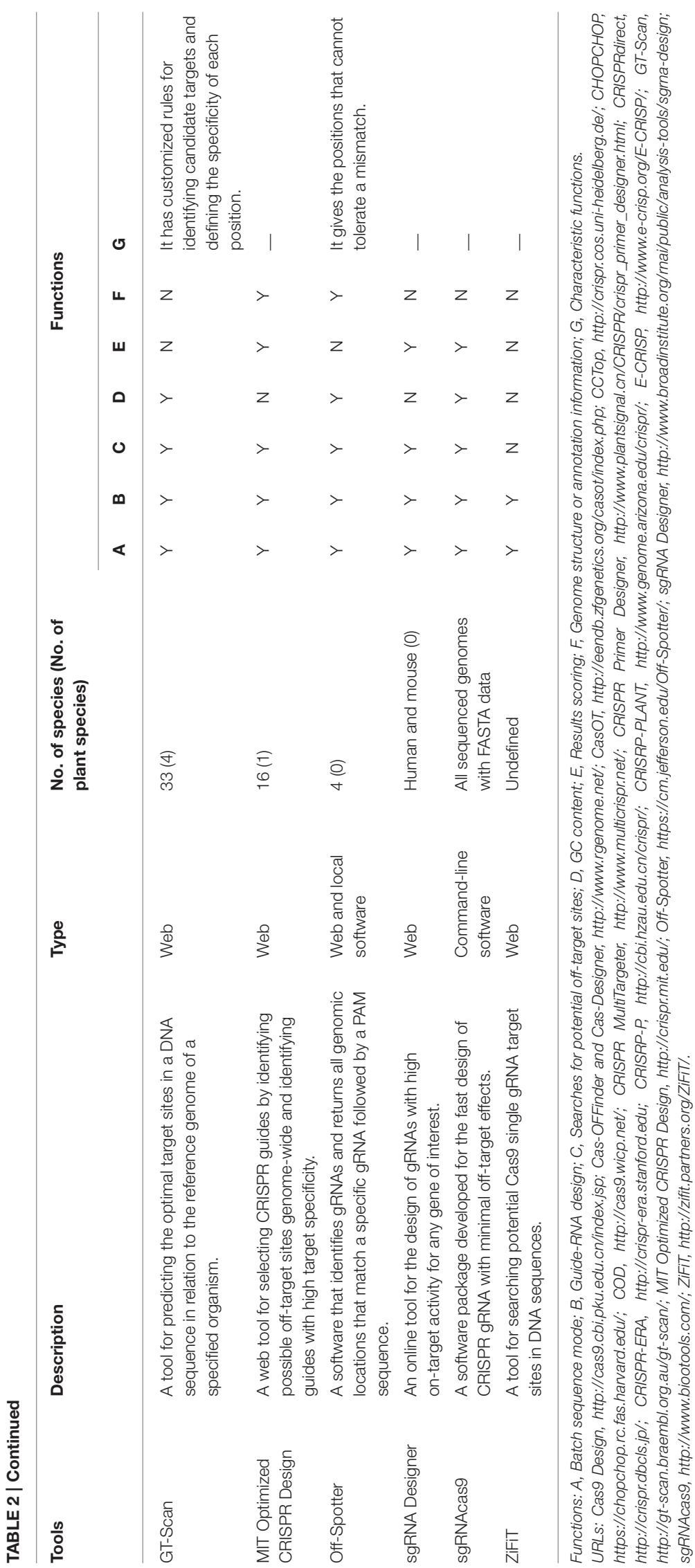


specific gRNAs could be designed to cover $\sim 90 \%$ of genes for seven out of eight plant species (Xie et al., 2014). This survey also explains why few off-target edits have been observed in plants.

Though highly specific gRNA might be designed in practice, the off-target activity of Cas9 reduces the number of its targetable sites. To overcome this pitfall, several methods have been developed to increase Cas9 targeting fidelity. Early efforts modified Cas9 into a nickase (Cas9 with either a D10A or H804A substitution and dCas9-FokI; Ran et al., 2013a; Guilinger et al., 2014; Tsai et al., 2014). These nickases need a pair of gRNAs to edit one site, thus needing $40 \mathrm{bp}$ of sequence, which reduces the possibility of off-target effects. Fusion of Cas9 with additional DNA-binding domains also reduces off-target editing (Bolukbasi et al., 2015). These modifications reduced the off-target risk, but also increased the complexity. Of note, shortening the gRNA spacer sequence to 17-18 nt increases targeting fidelity (Fu et al., 2014). Recently, the off-target problem was addressed by a more elegant approach. New Cas9 variants that are intolerant to any number of mismatches have been engineered by substituting 3-4 amino acids in Cas9 (Table 1; Kleinstiver et al., 2016). These high fidelity Cas 9 variants could be immediately adopted to address the off-target editing issue in plants.

\section{Cas9 VARIANTS AND ORTHOLOGS FOR GENOME ENGINEERING}

Besides off-target issues, the PAM requirement also restrains the Cas 9 targeting range, even though the PAM sequence of $5^{\prime}$-NGG$3^{\prime}$ appears at high frequency in genomes (5-12 times in every 100 bp for model plant species; Xie et al., 2014). To bypass the PAM limitation, a number of Cas9 orthologs from type II CRISPRCas systems were characterized and engineered for genome editing (summarized in Table 1). These Cas9 orthologs recognize different PAM sequences and are smaller than the commonly used S. pyogenes Cas9, providing useful alternatives for RNAguided genome engineering. Among these Cas9 orthologs, Cpf1 and FnCas9 recognize a PAM sequence of $5^{\prime}-\mathrm{TTN}-3^{\prime}$ and $5^{\prime}$-YG$3^{\prime}$, respectively (Ran et al., 2015; Hirano et al., 2016), which is present in genomes at an equal or higher frequency than $5^{\prime}$-NGG$3^{\prime}$. Because the CRISPR/Cas system is a general immunity system in bacteria and archaea, more Cas9 orthologs and other types of Cas with different PAM specificities will probably be found and engineered for genome editing in the near future.

Of note, the PAM specificities of Cas9 can be modified into other sequences by substituting several amino acid residues in the PAM-binding domain (Kleinstiver et al., 2015b). These new Cas9 variants can recognize a broad range of PAM sequences including $5^{\prime}$-NAGN-3', $5^{\prime}$-NGCG-3', and $5^{\prime}$-NGNG-3'. These improvements allow the commonly used S. pyogenes Cas 9 to edit almost any genomic site. After adoption of these Cas9 orthologs or variants, the PAM sequence restraint will be removed.

\section{BIOINFORMATIC TOOLS FOR CRISPR/Cas9 APPLICATIONS}

Many bioinformatic tools have been developed to facilitate Cas9-mediated genome editing (Table 2). These online platforms enable the design of specific gRNAs, predict the off-target sites of given gRNAs, and include other useful functions (e.g., assessing restriction enzyme cut sites; Table 2). Among these tools, only CRISPR-PLANT and CRISPR-P are specifically designed for Cas9-mediated plant genome editing. CRISPR-PLANT has a genome-wide survey of highly specific gRNAs in eight plant species and supports restriction enzyme analysis of target sites (Xie et al., 2014). CRISPR-P facilitates gRNA design for almost all plant species whose genome sequence is available and also provides off-target site analysis and restriction enzyme sequence analysis (Lei et al., 2014).

The rapid development of CRISPR/Cas9 technologies and their broad use in plants demand that more functions be integrated into these online tools. For plant biologists who are not familiar with genome sequence analysis, a plug-and-play online program would help them design a CRISPR/Cas9 experiment. In addition, such bioinformatics platforms should incorporate gRNA design for different Cas9 proteins with variable PAM specificities, predict the on-target editing efficiency and off-target risk of gRNAs, and assist in selecting the best target sites for Cas9 from tens of candidates. Besides, many important crops are polyploidy or outcrossing species, the sequence variation between different alleles should be considered for gRNA design. Because CRISPR/Cas9 genome editing is emerging as a general tool, a platform that integrates Cas9/gRNA design tools with genome annotation data would further facilitate genome-engineering experiments in basic research and crop breeding.

\section{CONCLUSIONS AND PERSPECTIVES}

Recent progress demonstrates that the CRISPR/Cas9 technology is becoming the ultimate molecular tool for genome engineering. Many of these Cas9-based technologies are being adopted in plants, and these tools will provide unprecedented insights into plant biology and enable us to improve crops with speed and accuracy through breeding. As genome-editing technology is widely used in plants, the safety of genome-edited crops is a matter of discussion in the plant community (Huang et al., 2016). Undoubtedly, the CRISPR/Cas9 technology is one of the most powerful tools in basic research and in crop genetic modification.

\section{AUTHOR CONTRIBUTIONS}

$\mathrm{KX}, \mathrm{YD}$, and HL collected references. KX, YD, and LC wrote and revised the manuscript. All authors read and approved the final manuscript.

\section{ACKNOWLEDGMENTS}

The present study was supported by grants from the National Natural Science Foundation of China (31571351) and the New Century Excellent Talents in University Program (NCET-130807) to LC, and grants from the Huazhong Agricultural University (2015RC009) and National Natural Foundation of China (31571374) to KX. We also thank Pablo Sanz Jimenez (College of Informatics, Huazhong Agricultural University) for advice on the language and grammar of this manuscript. 


\section{REFERENCES}

Baltes, N. J., Gil-Humanes, J., Cermak, T., Atkins, P. A., and Voytas, D. F. (2014). DNA replicons for plant genome engineering. Plant Cell 26, 151-163. doi: 10.1105/tpc.113.119792

Boch, J., Scholze, H., Schornack, S., Landgraf, A., Hahn, S., Kay, S., et al. (2009). Breaking the code of DNA binding specificity of TAL-type III effectors. Science 326, 1509-1512. doi: 10.1126/science.1178811

Bolukbasi, M. F., Gupta, A., Oikemus, S., Derr, A. G., Garber, M., Brodsky, M. H., et al. (2015). DNA-binding-domain fusions enhance the targeting range and precision of Cas9. Nat. Methods 12, 1150-1156. doi: 10.1038/nmeth.3624

Brooks, C., Nekrasov, V., Lippman, Z. B., and Van Eck, J. (2014). Efficient gene editing in tomato in the first generation using the clustered regularly interspaced short palindromic repeats/CRISPR-associated9 system. Plant Physiol. 166, 1292-1297. doi: 10.1104/pp.114.247577

Chavez, A., Scheiman, J., Vora, S., Pruitt, B. W., Tuttle, M., Iyer, E. P. R., et al. (2015). Highly efficient Cas9-mediated transcriptional programming. Nat. Methods 12, 326-328. doi: 10.1038/nmeth.3312

Cheng, A. W., Wang, H., Yang, H., Shi, L., Katz, Y., Theunissen, T. W., et al. (2013). Multiplexed activation of endogenous genes by CRISPR-on, an RNA-guided transcriptional activator system. Cell Res. 23, 1163-1171. doi: 10.1038/cr.2013.122

Cong, L., Ran, F. A., Cox, D., Lin, S., Barretto, R., Habib, N., et al. (2013). Multiplex genome engineering using CRISPR/Cas systems. Science 339, 819-823. doi: $10.1126 /$ science. 1231143

Cradick, T. J., Fine, E. J., Antico, C. J., and Bao, G. (2013). CRISPR/Cas9 systems targeting beta-globin and CCR5 genes have substantial off-target activity. Nucleic Acids Res. 41, 9584-9592. doi: 10.1093/nar/gkt714

Davis, K. M., Pattanayak, V., Thompson, D. B., Zuris, J. A., and Liu, D. R. (2015). Small molecule-triggered Cas9 protein with improved genome-editing specificity. Nat. Chem. Biol. 11, 316-318. doi: 10.1038/nchembio.1793

Deveau, H., Barrangou, R., Garneau, J. E., Labonte, J., Fremaux, C., Boyaval, P., et al. (2008). Phage response to CRISPR-encoded resistance in Streptococcus thermophilus. J. Bacteriol. 190, 1390-1400. doi: 10.1128/JB.01412-07

Doudna, J. A., and Charpentier, E. (2014). Genome editing. The new frontier of genome engineering with CRISPR-Cas9. Science 346:1258096. doi: 10.1126/science. 1258096

Fan, D., Liu, T., Li, C., Jiao, B., Li, S., Hou, Y., et al. (2015). Efficient CRISPR/Cas9mediated targeted mutagenesis in Populus in the first generation. Sci. Rep. 5:12217. doi: 10.1038/srep12217

Feng, Z., Mao, Y., Xu, N., Zhang, B., Wei, P., Yang, D. L., et al. (2014). Multigeneration analysis reveals the inheritance, specificity, and patterns of CRISPR/Cas-induced gene modifications in Arabidopsis. Proc. Natl. Acad. Sci. U.S.A. 111, 4632-4637. doi: 10.1073/pnas.1400822111

Feng, Z., Zhang, B., Ding, W., Liu, X., Yang, D. L., Wei, P., et al. (2013). Efficient genome editing in plants using a CRISPR/Cas system. Cell Res. 23, 1229-1232. doi: $10.1038 / \mathrm{cr} .2013 .114$

Fineran, P. C., and Charpentier, E. (2012). Memory of viral infections by CRISPRCas adaptive immune systems: acquisition of new information. Virology 434, 202-209. doi: 10.1016/j.virol.2012.10.003

Fu, Y., Foden, J. A., Khayter, C., Maeder, M. L., Reyon, D., Joung, J. K., et al. (2013). High-frequency off-target mutagenesis induced by CRISPR-Cas nucleases in human cells. Nat. Biotechnol. 31, 822-826. doi: 10.1038/nbt.2623

Fu, Y., Sander, J. D., Reyon, D., Cascio, V. M., and Joung, J. K. (2014). Improving CRISPR-Cas nuclease specificity using truncated guide RNAs. Nat. Biotechnol. 32, 279-284. doi: 10.1038/nbt.2808

Gao, Y., and Zhao, Y. (2014). Self-processing of ribozyme-flanked RNAs into guide RNAs in vitro and in vivo for CRISPR-mediated genome editing. J. Integr. Plant Biol. 56, 343-349. doi: 10.1111/jipb.12152

Gasiunas, G., Barrangou, R., Horvath, P., and Siksnys, V. (2012). Cas9-crRNA ribonucleoprotein complex mediates specific DNA cleavage for adaptive immunity in bacteria. Proc. Natl. Acad. Sci. U.S.A. 109, E2579-E2586. doi: 10.1073/pnas.1208507109

Gilbert, L. A., Larson, M. H., Morsut, L., Liu, Z., Brar, G. A., Torres, S. E., et al. (2013). CRISPR-mediated modular RNA-guided regulation of transcription in eukaryotes. Cell 154, 442-451. doi: 10.1016/j.cell.2013.06.044

Guilinger, J. P., Thompson, D. B., and Liu, D. R. (2014). Fusion of catalytically inactive Cas9 to FokI nuclease improves the specificity of genome modification. Nat. Biotechnol. 32, 577-582. doi: 10.1038/ nbt.2909

Hilton, I. B., D'ippolito, A. M., Vockley, C. M., Thakore, P. I., Crawford, G. E., Reddy, T. E., et al. (2015). Epigenome editing by a CRISPR-Cas9based acetyltransferase activates genes from promoters and enhancers. Nat. Biotechnol. 33, 510-517. doi: 10.1038/nbt.3199

Hirano, H., Gootenberg, J. S., Horii, T., Abudayyeh, O. O., Kimura, M., Hsu, P. D., et al. (2016). Structure and Engineering of Francisella novicida Cas9. Cell 164, 950-961. doi: 10.1016/j.cell.2016.01.039

Hou, Z., Zhang, Y., Propson, N. E., Howden, S. E., Chu, L. F., Sontheimer, E. J., et al. (2013). Efficient genome engineering in human pluripotent stem cells using Cas9 from Neisseria meningitidis. Proc. Natl. Acad. Sci. U.S.A. 110, 15644-15649. doi: 10.1073/pnas.1313587110

Hsu, P. D., Lander, E. S., and Zhang, F. (2014). Development and applications of CRISPR-Cas9 for genome engineering. Cell 157, 1262-1278. doi: 10.1016/j.cell.2014.05.010

Hsu, P. D., Scott, D. A., Weinstein, J. A., Ran, F. A., Konermann, S., Agarwala, V., et al. (2013). DNA targeting specificity of RNA-guided Cas9 nucleases. Nat. Biotechnol. 31, 827-832. doi: 10.1038/nbt.2647

Huang, S., Weigel, D., Beachy, R. N., and Li, J. (2016). A proposed regulatory framework for genome-edited crops. Nat. Genet. 48, 109-111. doi: 10.1038/ng.3484

Hwang, W. Y., Fu, Y., Reyon, D., Maeder, M. L., Tsai, S. Q., Sander, J. D., et al. (2013). Efficient genome editing in zebrafish using a CRISPR-Cas system. Nat. Biotechnol. 31, 227-229. doi: 10.1038/nbt.2501

Hyun, Y., Kim, J., Cho, S. W., Choi, Y., Kim, J. S., and Coupland, G. (2015). Site-directed mutagenesis in Arabidopsis thaliana using dividing tissue-targeted RGEN of the CRISPR/Cas system to generate heritable null alleles. Planta 241 271-284. doi: 10.1007/s00425-014-2180-5

Jacobs, T. B., LaFayette, P. R., Schmitz, R. J., and Parrott, W. A. (2015). Targeted genome modifications in soybean with CRISPR/Cas9. BMC Biotechnol. 15:16. doi: 10.1186/s12896-015-0131-2

Jia, H., and Wang, N. (2014). Targeted genome editing of sweet orange using Cas9/sgRNA. PLoS ONE 9:e93806. doi: 10.1371/journal.pone.0093806

Jiang, W., Bikard, D., Cox, D., Zhang, F., and Marraffini, L. A. (2013a). RNA-guided editing of bacterial genomes using CRISPR-Cas systems. Nat. Biotechnol. 31, 233-239. doi: 10.1038/nbt.2508

Jiang, W., Zhou, H., Bi, H., Fromm, M., Yang, B., and Weeks, D. P. (2013b). Demonstration of CRISPR/Cas9/sgRNA-mediated targeted gene modification in Arabidopsis, tobacco, sorghum and rice. Nucleic Acids Res. 41, e188. doi: $10.1093 /$ nar/gkt780

Jinek, M., Chylinski, K., Fonfara, I., Hauer, M., Doudna, J. A., and Charpentier, E. (2012). A programmable dual-RNA-guided DNA endonuclease in adaptive bacterial immunity. Science 337, 816-821. doi: 10.1126/science.1225829

Jinek, M., East, A., Cheng, A., Lin, S., Ma, E., and Doudna, J. (2013). RNA-programmed genome editing in human cells. Elife 2:e00471. doi: 10.7554/eLife.00471

Kim, D., Bae, S., Park, J., Kim, E., Kim, S., Yu, H. R., et al. (2015). Digenome-seq: genome-wide profiling of CRISPR-Cas9 off-target effects in human cells. Nat. Methods 12, 237-243. doi: 10.1038/nmeth.3284

Kim, S., Kim, D., Cho, S. W., Kim, J., and Kim, J. S. (2014). Highly efficient RNA-guided genome editing in human cells via delivery of purified Cas9 ribonucleoproteins. Genome Res. 24, 1012-1019. doi: 10.1101/gr.171322.113

Kleinstiver, B. P., Pattanayak, V., Prew, M. S., Tsai, S. Q., Nguyen, N. T., Zheng, Z., et al. (2016). High-fidelity CRISPR-Cas9 nucleases with no detectable genome-wide off-target effects. Nature 529, 490-495. doi: 10.1038/nature 16526

Kleinstiver, B. P., Prew, M. S., Tsai, S. Q., Nguyen, N. T., Topkar, V. V., Zheng, Z., et al. (2015a). Broadening the targeting range of Staphylococcus aureus CRISPR-Cas9 by modifying PAM recognition. Nat. Biotechnol. 33, 1293-1298. doi: $10.1038 /$ nbt.3404

Kleinstiver, B. P., Prew, M. S., Tsai, S. Q., Topkar, V. V., Nguyen, N. T., Zheng, Z., et al. (2015b). Engineered CRISPR-Cas9 nucleases with altered PAM specificities. Nature 523, 481-485. doi: 10.1038/nature14592

Konermann, S., Brigham, M. D., Trevino, A. E., Joung, J., Abudayyeh, O. O., Barcena, C., et al. (2015). Genome-scale transcriptional activation by an engineered CRISPR-Cas9 complex. Nature 517, 583-588. doi: 10.1038 /nature 14136 
Kuhn, J., and Binder, S. (2002). RT-PCR analysis of $5^{\prime}$ to $3^{\prime}$-end-ligated mRNAs identifies the extremities of cox 2 transcripts in pea mitochondria. Nucleic Acids Res. 30, 439-446. doi: 10.1093/nar/30.2.439

Kuscu, C., Arslan, S., Singh, R., Thorpe, J., and Adli, M. (2014). Genomewide analysis reveals characteristics of off-target sites bound by the Cas9 endonuclease. Nat. Biotechnol. 32, 677-683. doi: 10.1038/nbt.2916

Lawrenson, T., Shorinola, O., Stacey, N., Li, C., Ostergaard, L., Patron, N., et al. (2015). Induction of targeted, heritable mutations in barley and Brassica oleracea using RNA-guided Cas9 nuclease. Genome Biol. 16, 258. doi: 10.1186/s13059-015-0826-7

Lee, C. M., Cradick, T. J., and Bao, G. (2016). The Neisseria meningitidis CRISPRCas9 system enables specific genome editing in mammalian cells. Mol. Ther. 24, 645-654. doi: 10.1038/mt.2016.8

Lei, Y., Lu, L., Liu, H. Y., Li, S., Xing, F., and Chen, L. L. (2014). CRISPR-P: a web tool for synthetic single-guide RNA design of CRISPR-system in plants. Mol. Plant 7, 1494-1496. doi: 10.1093/mp/ssu044

Li, J. F., Norville, J. E., Aach, J., McCormack, M., Zhang, D., Bush, J., et al. (2013). Multiplex and homologous recombination-mediated genome editing in Arabidopsis and Nicotiana benthamiana using guide RNA and Cas9. Nat. Biotechnol. 31, 688-691. doi: 10.1038/nbt.2654

Li, Z., Liu, Z. B., Xing, A., Moon, B. P., Koellhoffer, J. P., Huang, L., et al. (2015). Cas9-guide RNA directed genome editing in Soybean. Plant Physiol. 169, 960-970. doi: 10.1104/pp.15.00783

Liang, Z., Zhang, K., Chen, K., and Gao, C. (2014). Targeted mutagenesis in Zea mays using TALENs and the CRISPR/Cas system. J. Genet. Genomics 41, 63-68. doi: 10.1016/j.jgg.2013.12.001

Lowder, L. G., Zhang, D., Baltes, N. J., Paul, J. W. III, Tang, X., Zheng, X., et al. (2015). A CRISPR/Cas9 toolbox for multiplexed plant genome editing and transcriptional regulation. Plant Physiol. 169, 971-985. doi: $10.1104 /$ pp. 15.00636

Ma, X., Zhang, Q., Zhu, Q., Liu, W., Chen, Y., Qiu, R., et al. (2015). A robust CRISPR/Cas9 system for convenient, high-efficiency multiplex genome editing in monocot and dicot plants. Mol. Plant 8, 1274-1284. doi: 10.1016/j.molp.2015.04.007

Maeder, M. L., Linder, S. J., Cascio, V. M., Fu, Y., Ho, Q. H., and Joung, J. K. (2013). CRISPR RNA-guided activation of endogenous human genes. Nat. Methods 10, 977-979. doi: 10.1038/nmeth.2598

Mali, P., Aach, J., Stranges, P. B., Esvelt, K. M., Moosburner, M., Kosuri, S., et al. (2013a). CAS9 transcriptional activators for target specificity screening and paired nickases for cooperative genome engineering. Nat. Biotechnol. 31, 833-838. doi: 10.1038/nbt.2675

Mali, P., Yang, L., Esvelt, K. M., Aach, J., Guell, M., Dicarlo, J. E., et al. (2013b). RNA-guided human genome engineering via Cas9. Science 339, 823-826. doi: $10.1126 /$ science. 1232033

Mao, Y., Zhang, H., Xu, N., Zhang, B., Gou, F., and Zhu, J. K. (2013). Application of the CRISPR-Cas system for efficient genome engineering in plants. Mol. Plant 6, 2008-2011. doi: $10.1093 / \mathrm{mp} / \mathrm{sst} 121$

Mao, Y., Zhang, Z., Feng, Z., Wei, P., Zhang, H., Botella, J. R., et al. (2016). Development of germ-line-specific CRISPR-Cas9 systems to improve the production of heritable gene modifications in Arabidopsis. Plant Biotechnol. J. 14, 519-532. doi: 10.1111/pbi.12468

Miao, J., Guo, D., Zhang, J., Huang, Q., Qin, G., Zhang, X., et al. (2013). Targeted mutagenesis in rice using CRISPR-Cas system. Cell Res. 23, 1233-1236. doi: $10.1038 / \mathrm{cr} .2013 .123$

Moscou, M. J., and Bogdanove, A. J. (2009). A simple cipher governs DNA recognition by TAL effectors. Science 326, 1501. doi: 10.1126/science.1178817

Nekrasov, V., Staskawicz, B., Weigel, D., Jones, J. D., and Kamoun, S. (2013). Targeted mutagenesis in the model plant Nicotiana benthamiana using Cas9 RNA-guided endonuclease. Nat. Biotechnol. 31, 691-693. doi: 10.1038/nbt.2655

Nissim, L., Perli, S. D., Fridkin, A., Perez-Pinera, P., and Lu, T. K. (2014). Multiplexed and programmable regulation of gene networks with an integrated RNA and CRISPR/Cas toolkit in human cells. Mol. Cell 54, 698-710. doi: 10.1016/j.molcel.2014.04.022

Pabo, C. O., Peisach, E., and Grant, R. A. (2001). Design and selection of novel Cys2His2 zinc finger proteins. Annu. Rev. Biochem. 70, 313-340. doi: 10.1146/annurev.biochem.70.1.313

Pattanayak, V., Lin, S., Guilinger, J. P., Ma, E., Doudna, J. A., and Liu, D. R. (2013). High-throughput profiling of off-target DNA cleavage reveals
RNA-programmed Cas9 nuclease specificity. Nat. Biotechnol. 31, 839-843. doi: $10.1038 /$ nbt.2673

Pennisi, E. (2013). The CRISPR craze. Science 341, 833-836. doi: $10.1126 /$ science.341.6148.833

Perez-Pinera, P., Kocak, D. D., Vockley, C. M., Adler, A. F., Kabadi, A. M., Polstein, L. R., et al. (2013). RNA-guided gene activation by CRISPRCas9-based transcription factors. Nat. Methods 10, 973-976. doi: 10.1038/ nmeth. 2600

Piatek, A., Ali, Z., Baazim, H., Li, L., Abulfaraj, A., Al-Shareef, S., et al. (2015). RNA-guided transcriptional regulation in planta via synthetic dCas9-based transcription factors. Plant Biotechnol. J. 13, 578-589. doi: 10.1111/pbi.12284

Qi, L., Haurwitz, R. E., Shao, W., Doudna, J. A., and Arkin, A. P. (2012). RNA processing enables predictable programming of gene expression. Nat. Biotechnol. 30, 1002-1006. doi: 10.1038/nbt.2355

Ramakrishna, S., Kwaku Dad, A. B., Beloor, J., Gopalappa, R., Lee, S. K., and Kim, H. (2014). Gene disruption by cell-penetrating peptide-mediated delivery of Cas 9 protein and guide RNA. Genome Res. 24, 1020-1027. doi: $10.1101 /$ gr. 171264.113

Ran, F. A., Cong, L., Yan, W. X., Scott, D. A., Gootenberg, J. S., Kriz, A. J., et al. (2015). In vivo genome editing using Staphylococcus aureus Cas9. Nature 520, 186-191. doi: 10.1038/nature14299

Ran, F. A., Hsu, P. D., Lin, C. Y., Gootenberg, J. S., Konermann, S., Trevino, A. E., et al. (2013a). Double nicking by RNA-guided CRISPR Cas9 for enhanced genome editing specificity. Cell 154, 1380-1389. doi: 10.1016/j.cell.2013.08.021

Ran, F. A., Hsu, P. D., Wright, J., Agarwala, V., Scott, D. A., and Zhang, F. (2013b). Genome engineering using the CRISPR-Cas9 system. Nat. Protoc. 8 , 2281-2308. doi: 10.1038/nprot.2013.143

Ray, A., and Langer, M. (2002). Homologous recombination: ends as the means. Trends Plant Sci. 7, 435-440. doi: 10.1016/S1360-1385(02)02327-0

Shan, Q., Wang, Y., Li, J., Zhang, Y., Chen, K., Liang, Z., et al. (2013). Targeted genome modification of crop plants using a CRISPR-Cas system. Nat. Biotechnol. 31, 686-688. doi: 10.1038/nbt.2650

Slaymaker, I. M., Gao, L., Zetsche, B., Scott, D. A., Yan, W. X., and Zhang, F. (2016). Rationally engineered Cas9 nucleases with improved specificity. Science 351, 84-88. doi: 10.1126/science.aad5227

Steinert, J., Schiml, S., Fauser, F., and Puchta, H. (2015). Highly efficient heritable plant genome engineering using Cas9 orthologues from Streptococcus thermophilus and Staphylococcus aureus. Plant J. 84, 1295-1305. doi: $10.1111 /$ tpj. 13078

Sun, Y., Zhang, X., Wu, C., He, Y., Ma, Y., Hou, H., et al. (2016). Engineering herbicide-resistant rice plants through CRISPR/Cas9-mediated homologous recombination of acetolactate synthase. Mol. Plant 9, 628-631. doi: 10.1016/j.molp.2016.01.001

Svitashev, S., Young, J. K., Schwartz, C., Gao, H., Falco, S. C., and Cigan, A. M. (2015). Targeted mutagenesis, precise gene editing, and site-specific gene insertion in maize using Cas9 and guide RNA. Plant Physiol. 169, 931-945. doi: 10.1104/pp.15.00793

Tsai, S. Q., Wyvekens, N., Khayter, C., Foden, J. A., Thapar, V., Reyon, D., et al. (2014). Dimeric CRISPR RNA-guided FokI nucleases for highly specific genome editing. Nat. Biotechnol. 32, 569-576. doi: 10.1038/nbt.2908

Tsai, S. Q., Zheng, Z., Nguyen, N. T., Liebers, M., Topkar, V. V., Thapar, V., et al. (2015). GUIDE-seq enables genome-wide profiling of off-target cleavage by CRISPR-Cas nucleases. Nat. Biotechnol. 33, 187-197. doi: 10.1038/nbt.3117

van der Oost, J. (2013). Molecular biology. New tool for genome surgery. Science 339, 768-770. doi: $10.1126 /$ science. 1234726

Voytas, D. F. (2013). Plant genome engineering with sequence-specific nucleases. Annu. Rev. Plant Biol. 64, 327-350. doi: 10.1146/annurev-arplant-042811105552

Wang, Y., Cheng, X., Shan, Q., Zhang, Y., Liu, J., Gao, C., et al. (2014). Simultaneous editing of three homoeoalleles in hexaploid bread wheat confers heritable resistance to powdery mildew. Nat. Biotechnol. 32, 947-951. doi: 10.1038/nbt.2969

Wang, Z. P., Xing, H. L., Dong, L., Zhang, H. Y., Han, C. Y., Wang, X. C., et al. (2015). Egg cell-specific promoter-controlled CRISPR/Cas9 efficiently generates homozygous mutants for multiple target genes in Arabidopsis in a single generation. Genome Biol. 16, 144. doi: 10.1186/s13059-015-0715-0

Woo, J. W., Kim, J., Kwon, S. I., Corvalan, C., Cho, S. W., Kim, H., et al. (2015). DNA-free genome editing in plants with preassembled 
CRISPR-Cas9 ribonucleoproteins. Nat. Biotechnol. 33, 1162-1164. doi: 10.1038/ nbt.3389

Xie, K., Minkenberg, B., and Yang, Y. (2015). Boosting CRISPR/Cas9 multiplex editing capability with the endogenous tRNA-processing system. Proc. Natl. Acad. Sci. U.S.A. 112, 3570-3575. doi: 10.1073/pnas.1420294112

Xie, K., and Yang, Y. (2013). RNA-guided genome editing in plants using a CRISPR-Cas system. Mol. Plant 6, 1975-1983. doi: 10.1093/mp/sst119

Xie, K., Zhang, J., and Yang, Y. (2014). Genome-wide prediction of highly specific guide RNA spacers for CRISPR-Cas9-mediated genome editing in model plants and major crops. Mol. Plant 7, 923-926. doi: 10.1093/mp/ssu009

Xing, H. L., Dong, L., Wang, Z. P., Zhang, H. Y., Han, C. Y., Liu, B., et al. (2014). A CRISPR/Cas9 toolkit for multiplex genome editing in plants. BMC Plant Biol. 14:327. doi: 10.1186/s12870-014-0327-y

Yan, L., Wei, S., Wu, Y., Hu, R., Li, H., Yang, W., et al. (2015). Highefficiency genome editing in Arabidopsis using YAO promoter-driven CRISPR/Cas9 system. Mol. Plant 8, 1820-1823. doi: 10.1016/j.molp.2015. 10.004

Zalatan, J. G., Lee, M. E., Almeida, R., Gilbert, L. A., Whitehead, E. H., La Russa, M., et al. (2015). Engineering complex synthetic transcriptional programs with CRISPR RNA scaffolds. Cell 160, 339-350. doi: 10.1016/j.cell.2014.11.052

Zhang, B., Yang, X., Yang, C., Li, M., and Guo, Y. (2016). Exploiting the CRISPR/Cas9 system for targeted genome mutagenesis in petunia. Sci. Rep. 6, 20315. doi: 10.1038/srep20315
Zhang, H., Zhang, J., Wei, P., Zhang, B., Gou, F., Feng, Z., et al. (2014). The CRISPR/Cas9 system produces specific and homozygous targeted gene editing in rice in one generation. Plant Biotechnol. J. 12, 797-807. doi: 10.1111/pbi.12200

Zhou, H., Liu, B., Weeks, D. P., Spalding, M. H., and Yang, B. (2014). Large chromosomal deletions and heritable small genetic changes induced by CRISPR/Cas9 in rice. Nucleic Acids Res. 42, 10903-10914. doi: 10.1093/nar/gku806

Zhou, X., Jacobs, T. B., Xue, L. J., Harding, S. A., and Tsai, C. J. (2015). Exploiting SNPs for biallelic CRISPR mutations in the outcrossing woody perennial Populus reveals 4-coumarate:CoA ligase specificity and redundancy. New Phytol. 208, 298-301. doi: 10.1111/nph.13470

Conflict of Interest Statement: The authors declare that the research was conducted in the absence of any commercial or financial relationships that could be construed as a potential conflict of interest.

Copyright (C) 2016 Ding, Li, Chen and Xie. This is an open-access article distributed under the terms of the Creative Commons Attribution License (CC BY). The use, distribution or reproduction in other forums is permitted, provided the original author(s) or licensor are credited and that the original publication in this journal is cited, in accordance with accepted academic practice. No use, distribution or reproduction is permitted which does not comply with these terms. 